\title{
Multicentre cohort study of acute cholecystitis management during the COVID-19 pandemic
}

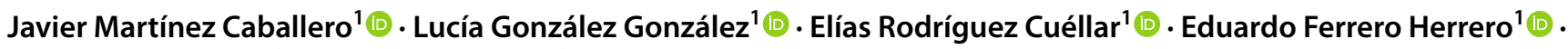 \\ Cristina Pérez Algar ${ }^{2}$. Victor Vaello Jodra ${ }^{\circledR}$. María Dolores Pérez Díaz ${ }^{3}$. Jana Dziakova ${ }^{4}$. \\ Rosario San Román Romanillos ${ }^{5} \cdot$ Marcello Di Martino $^{6}$ (1) - Ángela de la Hoz Rodríguez ${ }^{6}$ (D) Mónica Galán Martín ${ }^{7}$ (i) . \\ Daniel Sánchez López ${ }^{8}$ (1) Mariana García Virosta ${ }^{8}$ (1) $\cdot$ Marta de la Fuente Bartolomé ${ }^{9}(\mathbb{D} \cdot$ María de \\ Mar Pardo de Lama ${ }^{10}$ - María Gutiérrez Samaniego ${ }^{11}$ (D) David Díaz Pérez ${ }^{11}$ (D) David Alias Jiménez ${ }^{12}$ (D) \\ Luis de Nicolás Navas ${ }^{13}$. Juan José Pérez Alegre ${ }^{13}$. Javier García-Quijada García ${ }^{14}$. Jenny Guevara-Martínez ${ }^{15}$ (1) \\ Arantxa Villadoniga ${ }^{15} \cdot$ Roberto Martínez Fernández ${ }^{16}$
}

Received: 13 October 2020 / Accepted: 26 February 2021 / Published online: 19 March 2021

(c) Springer-Verlag GmbH Germany, part of Springer Nature 2021

\begin{abstract}
Purpose To analyse acute cholecystitis (AC) management during the first pandemic outbreak after the recommendations given by the surgical societies estimating: morbidity, length of hospital stay, mortality and hospital-acquired SARS-CoV-2 infection rate.

Methods Multicentre-combined (retrospective-prospective) cohort study with AC patients in the Community of Madrid between 1st March and 30th May 2020. 257 AC patients were involved in 16 public hospital. Multivariant binomial logistic regression (MBLR) was applied to mortality.

Results Of COVID-19 patients, 30 were diagnosed at admission and 12 patients were diagnosed during de admission or 30 days after discharge. In non-COVID-19 patients, antibiotic therapy was received in $61.3 \%$ of grade I AC and $40.6 \%$ of grade II AC. 52.4\% of grade III AC were treated with percutaneous drainage (PD). Median hospital stay was 5 [3-8] days, which was higher in the non-surgical treatment group with 7.51 days $(p<0.001)$ and a $3.25 \%$ of mortality rate $(p<0.21)$. 93.3\% of patients with SARS-CoV-2 infection at admission were treated with non-surgical treatment $(p=0.03)$, median hospital stay was 11.0 [7.5-27.5] days $(p<0.001)$ with a $7.5 \%$ of mortality rate $(p>0.05)$. In patients with hospital-acquired SARSCoV-2 infection, $91.7 \%$ of grade I-II AC were treated with non-surgical treatment $(p=0.037)$, with a median hospital stay of 16 [4-21] days and a $18.2 \%$ mortality rate $(p>0.05)$. Hospital-acquired infection risk when hospital stay is $>7$ days is OR 4.7, CI 95\% (1.3-16.6), $p=0.009$. COVID-19 mortality rate was $11.9 \%$, AC severity adjusted OR 5.64 (CI 95\% 1.417-22.64). In MBLR analysis, age (OR 1.15, CI 95\% 1.02-1.31), SARS-CoV-2 infection (OR 14.49, CI 95\% 1.33-157.81), conservative treatment failure (OR 8.2, CI 95\% 1.34-50.49) and AC severity were associated with an increased odd of mortality.

Conclusion In our population, during COVID-19 pandemic, there was an increase of non-surgical treatment which was accompanied by an increase of conservative treatment failure, morbidity and hospital stay length which may have led to an increased risk hospital-acquired SARS-CoV-2 infection. Age, SARS-CoV-2 infection, AC severity and conservative treatment failure were mortality risk factors.
\end{abstract}

Keywords Acute cholecystitis · COVID-19 · Hospital-acquired infection · SARS-CoV-2 · Cholecystectomy

\section{Abbreviations}

AC Acute cholecystitis

ICU Intensive care unit
PD Percutaneous drainage

RT-PCR Reverse transcriptase-polymerase chain reaction

Javier Martínez Caballero

j.mtnezcaballero90@gmail.com

Extended author information available on the last page of the article 


\section{Introduction}

The pandemic caused by the novel coronavirus SARSCoV-2 has had a great impact worldwide, especially in our national healthcare system, where the Community of Madrid has been the most affected area. The pandemic have had a remarkable impact on surgical hospital emergencies, with an approximately 50\% decrease in emergency surgical interventions [1]. In addition, $80 \%$ of the surgical services have modified the regular management of patients [2], 70\% have altered their work schedule and the most of the surgeons on payroll were reallocated to non-surgical services [1].

With regard to potentially surgical pathologies, during the pandemic surgeons must consider available resources (available hospital beds and ICU beds), operating rooms availability (since a great number of them were re-converted into rooms for critical patients), as well as expected hospital stays and potential infection risk for patients and healthcare workers during the admission process. Early cholecystectomy is the gold standard for treatment of AC, also laparoscopic approach should initially be attempted except absolute contraindications [3, 4]. Particularly, early cholecystectomy causes a shorter hospital stay and fewer complications than late cholecystectomy. Some European guidelines and publications have recommended non-surgical management of non-severe AC in this context $[2,5]$. Our main hypothesis is that non-surgical AC treatment of non-COVID-19 patients might increase the risk of hospital-acquired infection, hospital stay length, morbidity and mortality.

In this paper, we describe and analyse $\mathrm{AC}$ management in 16 hospitals in the Community of Madrid, during the higher incidence period of the pandemic, with the objective of drawing conclusions which helps to face future health crises in the emergency surgery filed. AC was chosen due to his high prevalence and for admitting both surgical and non-surgical treatment.

\section{Materials and methods}

We conducted a multicenter, observational, combined cohort study (retrospective-prospective). The period of data collection began on 1st March to 30th May 2020. 16 public hospitals in the Community of Madrid (Spain) were involved.

Inclusion criteria included (1) patients over 18 years and (2) hospital admission due to AC diagnosed at the emergency room or during hospitalisation by imaging test (ultrasounds, computerised tomography or nuclear magnetism resonance.
Exclusion criteria included (1) patients under 18 years, (2) acute pancreatitis with or without AC, (3) acute cholangitis, and (4) acute alithiasic cholecystitis.

The patient's inclusion in the study began on 1st April 2020, and it was followed 30-day after hospital discharge. The following up period ended on 30th June 2020 with 257 patients involved.

As regards AC, the following were analysed: diagnosis date, severity and treatment modality (open or laparoscopy cholecystectomy, percutaneous drainage (PD) or antibiotic therapy), conservative treatment failure, medical and postoperative complications (Clavien-Dindo Classification) [6], length of ICU stay, hospital stay length and 30-day mortality.

As regards COVID-19, the following were analysed: type of diagnosis (clinical, radiological or microbiological by reverse transcriptase-polymerase chain reaction (RT-PCR)), timing of diagnosis and severity of SARS-CoV-2 infection, length of ICU stay, AC treatment conditioning, patient operability, ICU/reanimation length stay, time from admission to COVID-19 diagnosis, and 30-day mortality.

AC diagnosis was considered since the moment of a conclusive imaging test and severity was determined based on the 2018 Tokyo guidelines [3]. Non-surgical treatment was considered as supportive treatment with antibiotic therapy; and conservative treatment failure as the need for cholecystectomy or PD after initiating non-surgical treatment. The timing of COVID-19 diagnosis was determined from the moment of positive RT-PCR testing or at admission with compatible clinical or radiological COVID-19 criteria. Hospital-acquired SARS-CoV-2 infection was defined as a positive RT-PCR testing or clinical and/or radiological COVID-19 diagnosis during admission, stay or 30 days after discharge. The definition and severity of SARS-CoV-2 infection was established according to the criteria set forth in the Health Ministry's technical report (3rd version, 19/03/2020) [7]. ICU admission depends on each hospital criteria and on patient needs.

This study is reported according to the STROBE guidelines for observational studies [8]. SPSS 23.0 software (IBM) was used for statistical analysis. Continuous data were tested for distribution, with non-normally distributed data presented as median with interquartile range and differences between groups were tested using the Mann-Whitney $U$. The $\chi^{2}$ and Fisher's exact tests were used for categorical data, which were expressed as percentage. Significance level of 5\% was set and $95 \%$ CI. To analyse whether AC severity may influence in mortality rate in SARS-CoV-2 patients homogeneity test (Breslow-Day and De Tarone) and the Cochran-Mantel-Haenszel $X^{2}$ test were performed.

Multivariant binomial logistic regression (MBLR) was performed to test the effect of age, BMI, diagnosis and timing of SARS-CoV-2 diagnosis, AC severity and conservative treatment failure in the mortality ( 30 days). Timing of 
COVID-19 diagnosis and BMI did not show significative differences, therefore, they were eliminated from the model and not included in the final analysis.

Ethical evaluation was not required by the 12 de Octubre University Hospital Ethics Committee. Data were collected by one or two researchers at each centre through REDCap online database. Each patient was assigned a personal ID to anonymise and protect their personal data. Only researchers at each centre had access to their own patient's data.

\section{Results}

16 hospitals in the Community of Madrid participated in the study with a population of 257 patients with AC (Table 1). The sample is made up of $146(56.8 \%)$ men and $111(43.2 \%)$ women. The demographic characteristics are shown in Table 2.
Table 1 Patients included by each hospital centre

\begin{tabular}{llll}
\hline Hospital centre & Acute cholecystitis & $\begin{array}{l}\text { COVID-19 infec- } \\
\text { tion at admission }\end{array}$ & $\begin{array}{l}\text { COVID-19 } \\
\text { hospital- acquired } \\
\text { infection }\end{array}$ \\
\hline Ramón y Cajal University Hospital (U.H) & $36(14 \%)$ & $1(3.3 \%)$ & $0(0 \%)$ \\
Gregorio Marañón U. H & $32(12.5 \%)$ & $7(23 \%)$ & $0(0 \%)$ \\
Clínico San Carlos U. H & $23(8.9 \%)$ & $3(10 \%)$ & $2(16.7 \%)$ \\
Príncipe de Asturias U. H & $23(8.9 \%)$ & $2(6.7 \%)$ & $1(8.3 \%)$ \\
12 de Octubre U. H & $22(8.9 \%)$ & $0(0 \%)$ & $3(25 \%)$ \\
La Princesa U. H & $21(8.6 \%)$ & $1(3.3 \%)$ & $1(8.3 \%)$ \\
Severo Ochoa U. H & $18(8.2 \%)$ & $5(16.7 \%)$ & $2(16.7 \%)$ \\
Infanta Sofía U. H & $15(5.8 \%)$ & $1(3.3 \%)$ & $0(0 \%)$ \\
Infanta Elena U. H & $14(5.4 \%)$ & $5(16.7 \%)$ & $0(0 \%)$ \\
Fundación Alcorcón U. H & $13(5.1 \%)$ & $0(0 \%)$ & $0(0 \%)$ \\
Torrejón U. H & $12(4.7 \%)$ & $1(3.3 \%)$ & $1(8.3 \%)$ \\
Rey Juan Carlos U. H & $9(3.5 \%)$ & $0(0 \%)$ & $0(0 \%)$ \\
Gómez Ulla Defense Central Hospital & $7(2.7 \%)$ & $0(0 \%)$ & $0(0 \%)$ \\
Getafe U.H & $5(1.9 \%)$ & $3(10 \%)$ & $2(16.7 \%)$ \\
La Paz U. H & $4(1.6 \%)$ & $1(3.3 \%)$ & $0(0 \%)$ \\
Tajo U. H & $3(0.8 \%)$ & $0(0 \%)$ & $0(0 \%)$ \\
Total & 257 & 30 & 12 \\
\hline
\end{tabular}

Table 2 Demographic characteristic of the population

\begin{tabular}{|c|c|c|c|c|c|}
\hline & Total $(n=257)$ & $\begin{array}{l}\text { non-COVID-19 infection } \\
(n=215)\end{array}$ & $\begin{array}{l}\text { COVID-19 infection } \\
\text { at admission }(n=30)\end{array}$ & $\begin{array}{l}\text { COVID-19 hospita- } \\
\text { lacquired infection } \\
(n=12)\end{array}$ & $p$ value \\
\hline Men/Women (\%) & $146(56.8 \%) / 111(43.2 \%)$ & $118(54.9 \%) / 97(45.1 \%)$ & $21(70 \%) / 9(30 \%)$ & $7(58.3 \%) / 5(41.2 \%)$ & 0.469 \\
\hline Age (median, IQR) & $69(52-80)$ & $68(50-80)$ & $71(60-80)$ & $83(65-87)$ & 0.017 \\
\hline No comorbidities (\%) & $96(37.4 \%)$ & $85(39.5 \%)$ & $10(33.3 \%)$ & $1(8.3 \%)$ & 0.098 \\
\hline Obesity (\%) & $83(7.9 \%)$ & $68(37.2 \%)$ & $9(42.9 \%)$ & $4(40 \%)$ & 0.880 \\
\hline Arterial hypertension (\%) & $115(44.7 \%)$ & $96(44.7 \%)$ & $12(40 \%)$ & $7(58.3 \%)$ & 0.281 \\
\hline Type 2 Diabetes mellitus (\%) & $68(26.5 \%)$ & $56(26 \%)$ & $8(26.7 \%)$ & $4(33.3 \%)$ & 0.666 \\
\hline $\begin{array}{l}\text { COPD/OSAS/ other chronic } \\
\text { lung diseases }(\%)\end{array}$ & $48(18.7 \%)$ & $39(18.1 \%)$ & $6(20.0 \%)$ & $3(25 \%)$ & 0.721 \\
\hline Heart disease $(\%)$ & $65(25.3 \%)$ & $48(22.3 \%)$ & $8(26.7 \%)$ & $9(75 \%)$ & 0.004 \\
\hline Immunosuppression (\%) & $6(2.3 \%)$ & $4(1.9 \%)$ & $1(3.3 \%)$ & $1(8.3 \%)$ & 0.492 \\
\hline Oncological (\%) & $16(6.2 \%)$ & $11(5.1 \%)$ & $4(13.3 \%)$ & $1(8.3 \%)$ & 0.651 \\
\hline $\begin{array}{l}\text { Dementia/cognitive impair- } \\
\text { ment }(\%)\end{array}$ & $14(5.4 \%)$ & $8(3.7 \%)$ & $3(10 \%)$ & $3(25 \%)$ & 0.209 \\
\hline $\begin{array}{l}\text { Institutionalized in nursing } \\
\text { home }(\%)\end{array}$ & $7(2.7 \%)$ & $4(1.9 \%)$ & $3(10 \%)$ & $0(0 \%)$ & 0.256 \\
\hline
\end{tabular}


42 patients (16.3\%) were diagnosed with $\mathrm{AC}$ and COVID19 simultaneously which were reported in 12 medical centres, of which $30(71.4 \%)$ patients were diagnosed with COVID-19 at admission and 12 (28.6\%) during hospital stay or 30 days after discharge (Table 2 and Fig. 1). Out of the 257 patients included, arterial hypertension (44.7\%), type 2 diabetes mellitus $(26.5 \%)$ and heart diseases $(25.6 \%)$ were the most frequent comorbidities.

According to the Tokyo guidelines, $43.6 \%$ of AC were grade I, $47.1 \%$ were grade II and $9.3 \%$ were grade III. Antibiotic therapy was given to $47.9 \%$, surgical procedure to $31.5 \%$ and PD was performed in $20.6 \%$ as initial therapy. Cholecystectomy was performed laparoscopically in $92.5 \%$ of cases and SARS-CoV-2 infection did not modify the approach in $98.8 \%$ of interventions.

Considering all the cohort of AC patients attended during the study period, $63.4 \%$ grade I AC were treated with antibiotic therapy and $27.6 \%$, with surgical approach. $40.5 \%$ of grade II AC received antibiotic therapy, surgical procedure was performed in $30.6 \%$, and $26.4 \%$ of patients were assigned to PD. In grade III, PD was the election treatment in $50 \%$ of patients while surgical procedure was performed in $14 \%$. The conservative treatment failure rate was $14.2 \%$, being more frequent in AC grade III $(42.8 \%, p=0.005)$. Overall postoperative complications rate was $26 \%$, being the most frequent Clavien-Dindo grade I $(70.1 \%, p<0.01)$, while severe complications (grade IV-V) were noticed in $14.9 \%$ of patients. Mean hospitalisation stay was 4.48 days in the surgical intervention group and 9.74 days in the conservative treatment group $(p=0.001)$. Mortality rate was $1.3 \%$ and $3.2 \%(p=0.075)$ in surgical and non-surgical treatment groups, respectively. Mortality after PD was significantly higher $(15.1 \%, p=0.001)$ compared to cholecystectomy (1.2\%) and antibiotic therapy (2.4\%).

As for the 215 patients without a COVID-19 diagnosis, $61.3 \%$ of grade I AC were treated with antibiotic therapy, $33.3 \%$ with cholecystectomy and PD was chosen in 5.4\%. In grade II AC, antibiotic therapy was elected in $40.6 \%$, in $38.6 \%$ cholecystectomy was performed and $20.8 \%$ required PD. As regards grade III AC, $52.4 \%$ were treated with PD while for $38.1 \%$ surgical procedure was performed. In $80.9 \%$ operated patients, they presented Clavien-Dindo grade I postoperative complication and severe complication (grade IV) was present in $6.4 \%$ of cases. One patient died postoperatively (grade V). Median hospital stay was 5 [3-8] days, being higher in non-surgical than in surgical group 7.51 and 3.94 days $(p<0.001)$, respectively. Overall mortality rate was $3.25 \%$, mortality rate between non-surgical and surgical group was $4.4 \%$ and $1.3 \%(p=0.21)$, respectively. In particular, mortality in patients treated with PD was $10.8 \%$ (4/37\%).

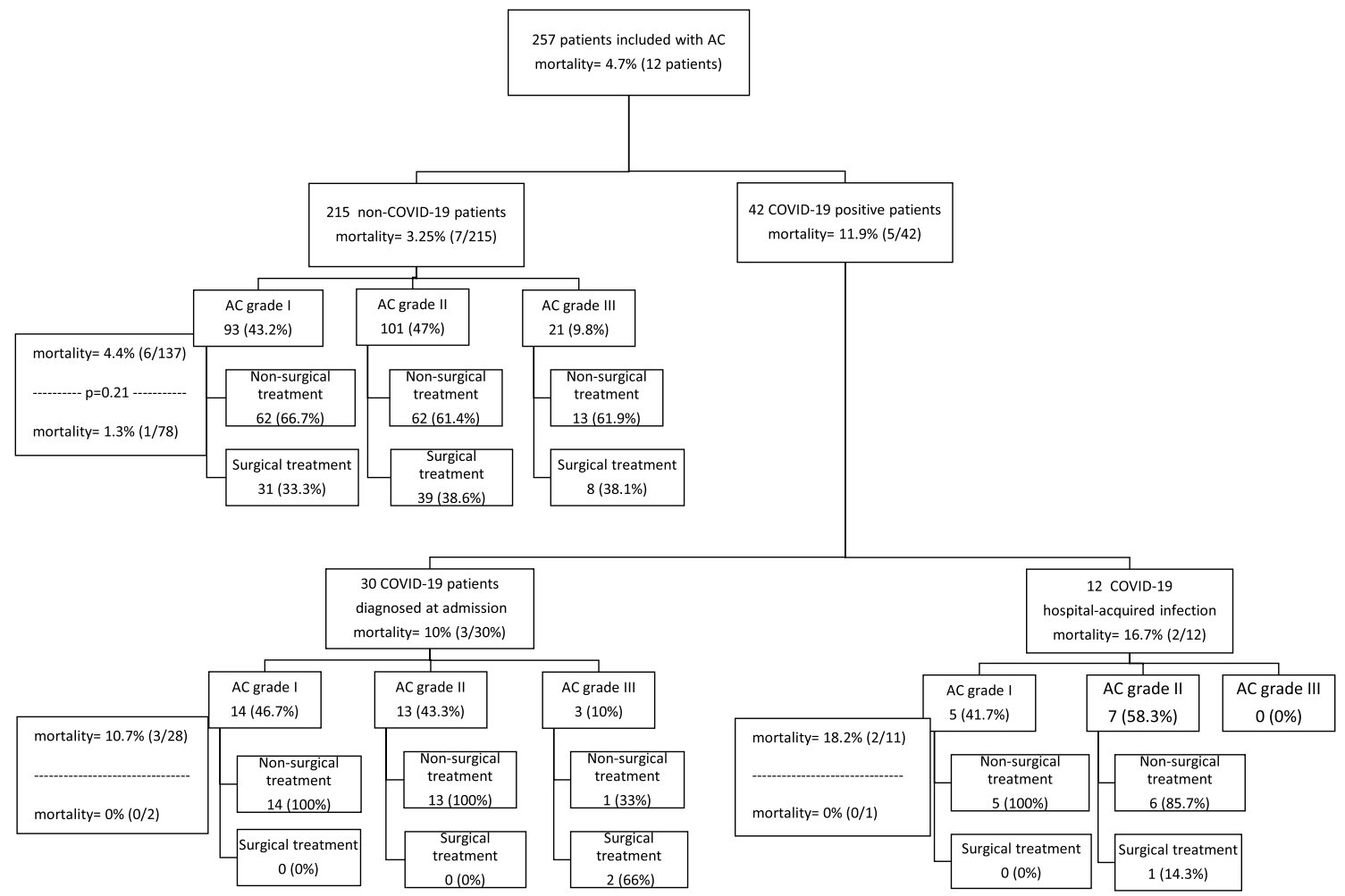

Fig. 1 Cohort patients' flowchart by COVID-19 diagnosis, timing of diagnosis (at admission or hospital-acquired infection) and mortality rates by treatment modality and $\mathrm{AC}$ severity 
In all the AC cohort, 42 patients presented SARS-CoV-2 infection. Diagnosis was based on clinics in $10.9 \%$, imaging tests in $11.3 \%$ or RT-PCR in $12.5 \%$ of all cases. SARSCoV-2 respiratory infection was asymptomatic in $29.3 \%$ of patients. On the other hand, $46.7 \%$ of symptomatic patients developed mild disease (non-complicated respiratory infection or mild pneumonia) and 53.3\% a severe disease (severe pneumonia, ADRS, sepsis or septic shock). No differences were observed in conservative treatment failure, worsening medical conditions neither mortality between symptomatic nor asymptomatic patients $(p>0.05)$. According to patients with severe disease $46.7 \%$ were admitted in ICU $(p=0.01)$. Mortality rate was $14.3 \%$ and $12.5 \%$ for mild and severe disease $(p>0.05)$, respectively.

Regarding the patients with AC and COVID-19 diagnosis present at admission, non-surgical treatment was performed in $93.3 \%$ and $6.7 \%$ were treated by surgery $(p=0.03)$, with a $16.7 \%$ of conservative treatment failure rate, and a $7.5 \%$ mortality rate $(p>0.05)$. Median hospital stay was 11.0 [7.5-27.5] days $(p<0.001)$, higher than for non-COVID-19 patients.

$85.7 \%$ of grade I AC were treated with antibiotic therapy, while PD was performed in $69.2 \%$ of grade II. Of these 30 patients with COVID-19 at admission, only 2 patients were surgically treated. However, only $6.7 \%$ were not considered fit for surgery and $20 \%$ were not considered suitable to ICU admission. 23\% of SARS-CoV-2-infected patients required ICU admission due to severe respiratory infection with a median ICU stay of 2 [1.75-2] days. 23\% were admitted in ICU at the moment of AC diagnosis.

As regards the patients with hospital-acquired SARSCoV-2 infection, 91.7\% of non-severe AC (grade I-II) received non-surgical treatment, of which $58.3 \%$ via antibiotic therapy. One patient was surgically treated by cholecystectomy (grade III AC). However, only one patient (8.3\%) was not considered fit for surgery. Conservative treatment failure rate was $45.5 \%$, especially, with worsening of previous medical conditions as cardiac arrest, acute kidney failure and acute respiratory failure. Non-surgical treatment mortality rate was $18.2 \%(p>0.005)$. Median hospital stay was 16 [4-21] days, while median time from admission to COVID19 diagnosis was 11 [8.2-21.75] days.

For the 227 negative RT-PCR patients at admission, the hospital-acquired infection rate has been estimated at $4.6 \%$. Risk of infection when hospital stay length is $>7$ days is OR 4.7 CI 95\% (1.3-16.6), $p=0.009$. Number needed to treat (NNT) by cholecystectomy to avoid one hospital-acquired SARS-CoV-2 infection is 16 (CI 95\% 9-77).

Complication description in the deceased patients is shown in Table 3. Four non-COVID-19 patients died due to

Table 3 Comorbidities and complications associated to AC and COVID-19 in deceased patients

\begin{tabular}{|c|c|c|c|c|c|c|c|c|c|}
\hline Gender & Age & BMI & Comorbidities & Timing of diagnosis & $\begin{array}{l}\text { Severity } \\
\text { respira- } \\
\text { tory }\end{array}$ & AC severity & Treatment & $\begin{array}{l}\text { Conserva- } \\
\text { tive treatment } \\
\text { failure }\end{array}$ & Complications \\
\hline M & 86 & $>30$ & AHT & Non-COVID & NA & Grade III & $\mathrm{AB}$ & Yes & Gallblader perforation \\
\hline $\mathrm{W}$ & 86 & $<30$ & $\begin{array}{l}\text { AHT, CPOD, onco- } \\
\text { logic }\end{array}$ & Non-COVID & NA & Grade II & $\mathrm{AB}$ & No & Biliar septic shock \\
\hline W & 75 & $>30$ & $\begin{array}{l}\text { AHT, DM2, cardi- } \\
\text { opathy, oncologic }\end{array}$ & Non-COVID & NA & Grade III & PD & Yes & Biliar septic shock \\
\hline M & 78 & $<30$ & AHT, DM2 & Non-COVID & NA & Grade III & PD & Yes & Gallblader perforation \\
\hline M & 84 & $<30$ & $\begin{array}{l}\text { AHT, DM2, cardi- } \\
\text { opathy }\end{array}$ & Non-COVID & NA & Grade II & Surgery & NA & $\begin{array}{l}\text { Non revascularizable } \\
\text { arterial trombosis }\end{array}$ \\
\hline W & 87 & $<30$ & $\begin{array}{l}\text { AHT, DM2, cardi- } \\
\text { opathy }\end{array}$ & Non-COVID & NA & Grade III & PD & Yes & Heart failure, AKD \\
\hline M & 91 & $<30$ & $\begin{array}{l}\text { AHT, DM2, cardi- } \\
\text { opathy }\end{array}$ & Non-COVID & NA & Grade III & PD & Yes & AKD, DIC \\
\hline W & 88 & $>30$ & AHT, DM2 & Admission & Severe & Grade I & PD & Yes & Respiratory distress \\
\hline M & 88 & $<30$ & $\begin{array}{l}\text { AHT, DM2, COPD, } \\
\text { cardiopathy }\end{array}$ & Admission & Severe & Grade II & PD & No & Respiratory distress \\
\hline M & 72 & $<30$ & AHT, DM2 & Admission & Severe & Grade III & PD & Yes & $\begin{array}{l}\text { Respiratory septic } \\
\text { shock }\end{array}$ \\
\hline M & 85 & $>30$ & $\begin{array}{l}\text { Cardiopathy, demen- } \\
\text { tia }\end{array}$ & Hospital & Mild & Grade II & $\mathrm{AB}$ & Yes & AKD \\
\hline W & 81 & $>30$ & $\begin{array}{l}\text { AHT, DM2, COPD, } \\
\text { cardiopathy }\end{array}$ & Hospital & Mild & Grade II & PD & No & Heart failure, AKD \\
\hline
\end{tabular}

$A B$ antibiotic therapy; $A H T$ arterial hypertension; $A K D$ acute kidney disease; $D I C$ disseminated intravascular coagulation; $D M 2$ diabetes mellitus type 2; $M$ man; $N A$ not apply; $P D$ percutaneous drainage; $W$ woman; 
AC complications (septic shock or gallbladder perforation) and three died due to medical complications. COVID-19 patients diagnosed at admission (3/12), regardless of AC severity, all developed severe respiratory disease by acute respiratory distress or respiratory septic shock that led to death. Finally, hospital-acquired infection patients developed a mild respiratory infection and died due to worsening of previous medical conditions.

COVID-19 overall mortality rate was $11.9 \%$, OR 4.05 (CI 95\% 1.2-13.3), $p=0.015$, higher than in nonCOVID-19 patients and AC severity adjusted OR 5.64 (CI 95\% 1.417-22.64). In patients with COVID-19 diagnosis at admission mortality rate was $10.3 \%$ and $16.7 \%$ in hospitalacquired infection (Table 4).

The MBLR was significative (omnibus test $<0.05$ ) and explains between $22.9 \%$ and $61.3 \%$ of the dependent variable (mortality 30 days) and classifies properly $96.6 \%$ of cases, therefore, the model can be considered acceptable (Table 5).

Compared to younger patients, the odds of death were higher in older patients $(B=0.16 ; p=0.024$; OR 1.17 (CI $95 \%$ 1.02-1.34). Patients with SARS-CoV-2 infection had significantly higher odds of death compared than nonCOVID-19 patients $(B=2.67 ; p=0.028$; OR 14.49 , CI $95 \%$ 1.33-157.80). About AC severity, grade I (OR 0.04 CI 95\%
$0.00-0.15)$ and grade II (OR 0.025, CI 95\% 0.02-0.34) were associated with significantly reduction of $4 \%$ and $2.5 \%$ in odds of death than grade III AC. Grade III AC was significative $(p=0.00)$ in the bivariant analysis. Conservative treatment failure was associated with a very increased odds of death in patients with $\mathrm{AC}(\mathrm{B}=2.106 ; p=0.023$; OR 8.2, CI 95\% 1.34-50.49).

\section{Discussion}

This study describes the AC management between 1st March and 30th May 2020 during the COVID-19 pandemic. In spite of the many surgical societies' recommendations about AC management, there is no clear evidence about AC management during this particular health crisis. This article aims to serve as a starting point to optimise $\mathrm{AC}$ management in future COVID-19 outbreaks.

During SARS-CoV-2 pandemic, it has been registered a $14 \%$ reduction in emergency hospital admission due to acute abdomen [9], as a $52.7 \%$ decrease of emergency surgical activity with a prevalence of $42 \%$ for acute peritonitis [10]. There was a general subjective perception of higher incidence and severity of AC during de COVID-19 outbreak,
Table 4 Outcomes according to the diagnosis and time of COVID-19 diagnosis

\begin{tabular}{llllc}
\hline & $\begin{array}{l}\text { non-COVID-19 } \\
\text { infection } \\
(n=215)\end{array}$ & $\begin{array}{l}\text { COVID-19 } \\
\text { at admission } \\
(n=30)\end{array}$ & $\begin{array}{l}\text { COVID-19 hospital } \\
\text { acquired infection } \\
(n=12)\end{array}$ & $p$ value \\
\hline $\begin{array}{l}\text { AC severity } \\
\text { Treatment }\end{array}$ & Grade II (47\%) & Grade I (46,7\%) & Grade II (41.5\%) & 0.796 \\
Surgical & $36.3 \%$ & $6.7 \%$ & $8.3 \%$ & 0.001 \\
Non-surgical & $63.7 \%$ & $93.3 \%$ & $91.7 \%$ & 0.009 \\
Failure of conservative treatment & $12.4 \%$ & $10.7 \%$ & $45.5 \%$ & - \\
Postoperative complications & $26 \%$ & - & - & 0.001 \\
Median hospital stay (days) & $5[3-8]$ & $11[7.5-21]$ & $16[4-21]$ & 0.034 \\
Mortality (30 days follow-up) & $3.2 \%$ & $10 \%$ & $16.7 \%$ & \\
\hline
\end{tabular}

Table 5 Multivariant binomial logistic regression analysis of mortality (30 days)

\begin{tabular}{|c|c|c|c|c|c|c|c|c|}
\hline & $B$ & $\begin{array}{l}\text { Standards } \\
\text { error }\end{array}$ & Wald & gl & $p$ value & $\operatorname{Exp}(B)$ & $\begin{array}{l}95 \% \text { CI } \\
\text { lower }\end{array}$ & 95\% CI higher \\
\hline Age & 0.159 & 0.070 & 5.120 & 1 & 0.024 & 1.172 & 1.021 & 1.344 \\
\hline $\begin{array}{l}\text { SARS- } \\
\text { CoV-2 } \\
\text { infection }\end{array}$ & 2.674 & 1.218 & 4.820 & 1 & 0.028 & 14.499 & 1.332 & 157.807 \\
\hline Grade I AC & -5.457 & 1.833 & 8.867 & 1 & 0.003 & .004 & .000 & 0.155 \\
\hline Grade II AC & -3.698 & 1.349 & 7.521 & 1 & 0.006 & .025 & .002 & 0.348 \\
\hline $\begin{array}{l}\text { Con- } \\
\text { servative } \\
\text { treatment } \\
\text { failure }\end{array}$ & 2.106 & 0.927 & 5.165 & 1 & 0.023 & 8.214 & 1.336 & 50.496 \\
\hline
\end{tabular}


grade II-II supposed $56.4 \%$ of AC episodes. It has been observed a decrease in $\mathrm{AC}$ diagnosis during the coincident period of higher prevalence of SARS-CoV-2 infections (Fig. 2). This could be related with the public measures that have been implemented to reduce the mobility, the fear of going to hospital due to contagion risk and the spontaneous resolution of uncomplicated biliary colic or mild AC episodes self-limited with symptomatic treatment at home. This would reduce the overall AC diagnosis and it would increase the severity of AC presentation.

Early cholecystectomy has been established as the treatment of choice for AC, as far as 10 days from the onset of symptoms, since it has shown shorter hospital stay length, lesser comorbidity and greater cost-effectiveness $[4,11$, 12]. During the COVID-19 outbreak, despite the COVID19 absence, non-surgical treatment was used in $66.7 \%$ and $61.4 \%$ of grade I and grade II AC, respectively. Only $25 \%$ of patients were surgically treated by cholecystectomy with a $26 \%$ postoperative complication rate, the most of them were mild (Clavien-Dindo grade I). However, type of treatment for AC (non-surgical or surgical) in non-COVID-19 patients did not show improvement in mortality rate (4.4 vs $1.3, p=0.21$ ).

For higher-risk patients, PD might be considered as an alternative choice of treatment [4]. In our series, PD mortality rate is $15 \%$, these results are similar to those published in other studies [13]. CHOCOLATE trial should be noted because it shows a higher median hospital stay [9 (6-19) days] with higher readmission rate, complications, reoperations due to biliary disease and mortality in the PD group [14]. In this sense, WSES in 2016 did not recommend PD as an alternative treatment to cholecystectomy due to a significantly higher mortality rate, between 4 and 50\% [4].
Some authors suggest that SARS-CoV-2 infection might be asymptomatic in $17-50 \%$ of cases $[15,16]$. Severe respiratory infection and ICU admission might be developed in $8-20 \%$ of cases, with a $14-66 \%$ mortality rate [16]. In our study, asymptomatic infection has been the most frequent presentation of the disease. AC patients with symptomatic patients developed frequently severe infection with $20 \%$ of ICU admission.

In patients with COVID-19 diagnosis at admission, nearly all centres chose antibiotic therapy or PD in grade I and grade II AC, respectively, and only two patients underwent surgery due to grade III AC. It seems that this choice did not depend on patient's operability, because only $6.7-8.3 \%$ of patients were classified as unfitting for surgery. On the other hand, higher infection risk for operating room staff, ICU and operating room availability as well as the extrapolation of poor postoperative outcomes published in elective surgery in SARS-CoV-2 patients [17-19] might have been crucial in the choice of non-surgical treatment as an initial approach.

Although evidence about postoperative complications in COVID-19 patients is limited, results have been sufficient for different surgical societies to recommend avoiding surgery for these patients. Doglietto et al. reported a higher rate of respiratory, haemorrhagic and thrombotic postoperative complications in COVID-19 patients [18]. Lei et al. published a study with 34 patients who developed respiratory symptoms after surgical intervention, considering that they were in incubating period at the moment of surgery. These authors reported a $20 \%$ mortality rate, a $44 \%$ of ICU admission and 32.4\% developed ADRS [17]. In a 1128 patients cohort study with COVID-19 diagnosis who underwent surgery, mortality rate was $25 \%$, being higher in emergency surgery [20].
Fig. 2 Acute cholecystitis and COVID-19 diagnoses evolution during study period

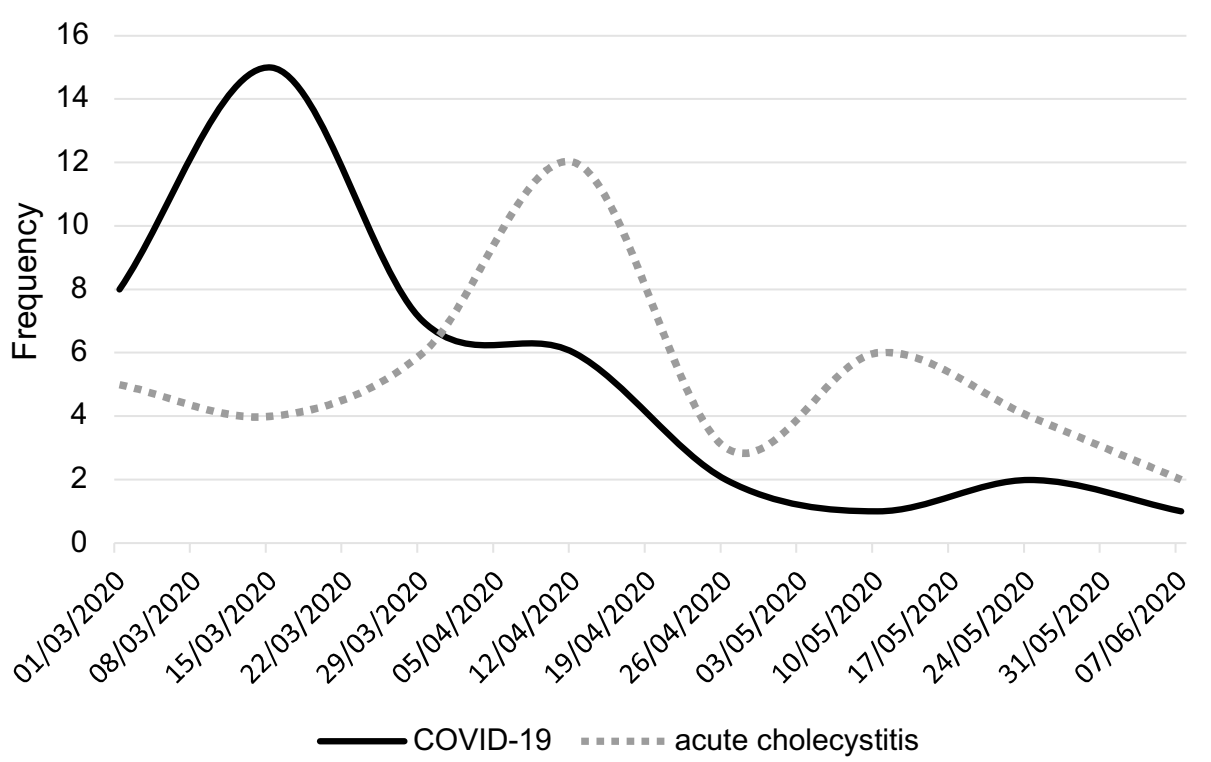


These results are consistent with the position statement of the Spanish Surgeons Association (Asociación Española de Cirujanos, AEC) and other societies [2, 5], who recommend "considering conservative treatment in uncomplicated AC assuming a higher readmission rate, higher conservative treatment failure and greater complexity in future cholecystectomy [...], as well as considering widening PD indications in COVID-19 patients during the pandemic outbreak" [2]. In addition, the high prevalence of severe respiratory diseases might favour the non-surgical treatment as a first choice given the risk of worsening respiratory condition after general anaesthesia, and to reduce contagion risk to health staff.

Subgroup of patients with hospital-acquired COVID19 infection $(n=12)$, without evidence of COVID-19 at admission (negative RT-PCR and asymptomatic), were diagnosed with SARS-CoV-2 infection during their hospital stay or 30 days after hospital discharge. That means a $4.6 \%$ hospital-acquired infection rate.

Antibiotic therapy was the treatment of choice for 91.7\% of grade I-II AC patients, with a median hospital stay length of 11 days from admission to COVID-19 diagnosis and a case mortality rate of $18.2 \%$, which is similar to other authors [17]. The choice of antibiotic therapy or PD in grade I-II AC patients is associated with higher conservative treatment failure rate, greater complications and longer hospital stay [14]. It is not possible to draw conclusions about mortality and surgical treatment in COVID19 patients because of the limited of surgery procedures performed $(n=3)$.

SARS-CoV-2 infection is a mortality risk factor [18]. Mortality rate may vary between 0.2 and $12.9 \%$ in 18 to $>80$-year-old patients, respectively; and $0.25-23.8 \%$ without comorbidities or with one comorbidity as chronic kidney disease [16]. In our population, SARS-CoV-2 infection was associated with fourfold increased odds of death (OR 4.05, CI 95\% 1.2-13.3), but when the mortality odds was adjusted by AC severity increased 5.64 times the odds of death. As adjusted OR was similar but higher than raw OR for mortality, AC might be an effect modifier variable increasing mortality odds in SARS-CoV-2 patients.

After the MBLR analysis, the relation of age, SARSCoV-2 infection, AC severity and conservative treatment failure mortality (30 days) in AC stands out. In our model, SARS-CoV-2 infection and conservative treatment failure showed the stronger association with mortality. Overall mortality in AC patients with COVID-19 at admission was $10 \%$, almost threefold in comparison to non-COVID-19 patients (3.5\%), but after MBLR, timing of SARS-CoV-2 diagnosis were not significative in mortality odds neither BMI. We must consider the small sample size of diagnosis at admission $(n=30)$ and hospital-acquired infection $(n=12)$ group that could lead to a loss of statistical power.
Based on available evidence, it seems appropriate to avoid or reduce surgical interventions in COVID-19 patients with AC to the essential cases. However, for non-COVID-19 patients with $\mathrm{AC}$, any treatment other than surgery increases hospital stay to 7.5 days compared to 3.9 days $(p<0.001)$, which contributes, on the one hand, to decreasing the available ICU beds and, on the other hand, to increasing the conservative treatment failure rate which might lead to a necessary PD or late cholecystectomy, with higher complications rate and a new increase in the length of hospital stay.

This paper shows that $>7$-day hospital stay increases the odds of SARS-CoV-2 infection by 4.7, CI 95\% (1.3-16.6). Longer hospital stay might favour contagion and nosocomial SARS-CoV-2 infection which might be severe and need ICU admission, especially in older patients and with associated comorbidities [21]. We encourage to enhance the preventive measures of health workers in the ward towards nonsurgically treated AC patients, to avoid contagion of SARSCoV-2 during admission. In addition, these patients who are generally unfit for surgery have multiple risk factors for mortality. Some authors warn that age and other risk factors for COVID-19 disease should not be considered sufficient to indicate alternative treatment except for real impracticability conditions for cholecystectomy [22].

We have conducted a multicentre study which allows to obtain the representative sample of the real situation in the Community of Madrid during the pandemic. The main limitation is that it is a combined prospective-retrospective study, where retrospective data were obtained by electronic medical record and the interpretation of these data might suppose a bias. In addition, it has been influenced by interindividual variability in clinical decisions and the high hospital occupation, ICU and operating rooms available at each centre in different phases of the pandemic. The choice of the most appropriate $\mathrm{AC}$ treatment by surgeons must be made considering patient characteristics, AC severity, available hospital resources, and the epidemiologic and healthcare centre resources context. Due to the relatively small sample size of COVID-19 group compared with non-COVID-19, this could lead to a type II error.

\section{Conclusions}

In our population, during COVID-19 pandemic, there was an increase of non-surgical treatment which was accompanied by an increase of conservative treatment failure, morbidity and hospital stay length which may have led to an increased risk hospital-acquired SARS-CoV-2 infection. Age, SARSCoV-2 infection, AC severity and conservative treatment failure were mortality risk factors. According to the results obtained, in our health system and in the pandemic context, early cholecystectomy might be carefully and individually 
assessed as the initial treatment in the absence of SARS$\mathrm{CoV}-2$ infection to try to reduce the risk of hospital-acquired infection.

Acknowledgements The authors are especially grateful to Carolina Varela Rodríguez from 12 de Octubre University Hospital's Quality Unit for her supervising of the methodology and to Manuel Juan Juan for his collaboration in translation tasks.

Funding This research was not supported by a particular or public statement.

\section{Declarations}

Conflict of interest There are no conflicts of interest.

Ethical approval Evaluation was not required after consulting the $12 \mathrm{de}$ Octubre University Hospital Research Ethics Committee.

\section{References}

1. Patriti A, Baiocchi GL, Catena F, Marini P, Catarci M, Beatrice DV, et al. Emergency general surgery in Italy during the COVID19 outbreak: First survey from the real life. World J Emerg Surg. 2020;15:15-36.

2. Aranda-Narváez JM, Tallón-Aguilar L, Pareja-Ciuró F, MartínMartín G, González-Sánchez AJ, Rey-Simó I, et al. Emergency surgery and trauma care during COVID-19 pandemic. Recommendations of the Spanish Association of Surgeons. Cir Esp. 2020;98:433-41.

3. Yokoe M, Hata J, Takada T, Strasberg SM, Asbun HJ, Wakabayashi G, et al. Tokyo Guidelines 2018: diagnostic criteria and severity grading of acute cholecystitis (with videos). J Hepatobiliary Pancreat Sci. 2018;25:41-54.

4. Ansaloni L, Pisano M, Coccolini F, Peitzmann AB, Fingerhut A, Catena F, et al. 2016 WSES guidelines on acute calculous cholecystitis. World J Emerg Surg. 2016;11:25.

5. de Simone B, Chouillard E, Di Saverio S, Pagani L, Sartelli M, Biffl WL, et al. Emergency surgery during the COVID-19 pandemic: What you need to know for practice. Ann R Coll Surg Engl. 2020;102:323-32.

6. Dindo D, Demartines NCP. Classification of surgical complications: a new proposal with evaluation in a cohort of 6336 patients and results of a survey. Ann Surg. 2005;240:205-13.

7. Luis M, Talía E, David S, Laura A, María E, Barrio I, et al. Documento técnico Manejo clínico del COVID-19: atención hospitalaria. Madrid, Spain. 2020;98:618-24.

8. Vandenbroucke JP, Von Elm E, Altman DG, Gøtzsche PC, Mulrow $\mathrm{CD}$, Pocock SJ, et al. Mejorar la comunicación de estudios observacionales en epidemiología (STROBE): explicación y elaboración. Gac Sanit. 2009;23:1-28.

9. Pérez-Rubio Á, Sebastián Tomás JC, Navarro-Martínez S, Gonzálvez GP, Torrecillas Meroño DG, Del Domingo PC.
Incidence of surgical abdominal emergencies during SARSCoV-2 pandemic. Cir Esp. 2020;98:618-24.

10. Álvarez GM, de Gortázar CS, Pascual MI, Rubio-Pérez I, Barragán SC, Álvarez PE, et al. SARS-CoV-2 pandemic on the activity and professionals of a General Surgery and Digestive Surgery Service in a tertiary hospital. Cir Esp. 2020;98:320-7.

11. Mayumi T, Okamoto K, Takada T, Strasberg SM, Solomkin JS, Schlossberg D, et al. Tokyo Guidelines 2018: management bundles for acute cholangitis and cholecystitis. J Hepatobiliary Pancreat Sci. 2018;25:96-100.

12. Zafar SN, Obirieze A, Adesibikan B, Cornwell EE, Fullum TM, Tran DD. Optimal time for early laparoscopic cholecystectomy for acute cholecystitis. JAMA Surg. 2015;150:129-36.

13. Loozen CS, Oor JE, van Ramshorst B, van Santvoort HC, Boerma D. Conservative treatment of acute cholecystitis: a systematic review and pooled analysis. Surg Endosc. 2017;31:504-15.

14. Loozen CS, Van Santvoort HC, Van Duijvendijk P, Besselink MG, Gouma DJ, Nieuwenhuijzen GA, et al. Laparoscopic cholecystectomy versus percutaneous catheter drainage for acute cholecystitis in high risk patients (CHOCOLATE): Multicentre randomised clinical trial. BMJ. 2018. https://doi.org/10.1136/bmj.k3965.

15. Esteban O-P, Katherine S-R, Lenin G-BMRN, Linda PG, Nikolaos CK, Claire M, Ana MGJ, Carlos B-O, Doménica C-R, Hugo S-SM, Luis U, Rasa Z, Naomi GALC. Clinical, molecular, and epidemiological characterization of the SARS-CoV-2 virus and the Coronavirus Disease 2019 (COVID-19), a comprehensive literature review. Diagn Microbiol Infect Dis. 2020;98:1-31.

16. Stawicki S, Jeanmonod R, Miller A, Paladino L, Gaieski D, Yaffee A, et al. The 2019-2020 novel coronavirus (severe acute respiratory syndrome coronavirus 2) pandemic: a joint american college of academic international medicine-world academic council of emergency medicine multidisciplinary COVID-19 working group consensus paper. J Glob Infect Dis. 2020;12:47-93.

17. Lei S, Jiang F, Su W, Chen C, Chen J, Mei W, et al. Clinical characteristics and outcomes of patients undergoing surgeries during the incubation period of COVID-19 infection. Clin Med. 2020;21:100331.

18. Doglietto F, Vezzoli M, Gheza F, Lussardi GL, Domenicucci M, Vecchiarelli L, et al. Factors associated with surgical mortality and complications among patients with and without coronavirus disease 2019 (COVID-19) in Italy. JAMA Surg. 2020;155:1-14.

19. Aminian A, Safari S, Razeghian-Jahromi A, Ghorbani M, Delaney CP. COVID-19 outbreak and surgical practice: unexpected fatality in perioperative period. Ann Surg. 2020;272:e27-9.

20. Nepogodiev D, Bhangu A, Glasbey JC, Li E, Omar OM, Simoes JF, et al. Mortality and pulmonary complications in patients undergoing surgery with perioperative SARS-CoV-2 infection: an international cohort study. Lancet. 2020;396:27-38.

21. Lee LYW, Cazier JB, Starkey T, Turnbull CD, Kerr R, Middleton G. COVID-19 mortality in patients with cancer on chemotherapy or other anticancer treatments: a prospective cohort study. Lancet. 2020;395:1919-26.

22. Campanile FC, Podda M, Arezzo A, Botteri E, Sartori A, Guerrieri M, et al. Acute cholecystitis during COVID-19 pandemic: A multisocietary position statement. World J Emerg Surg. 2020. https://doi.org/10.1186/s13017-020-00317-0. 


\section{Authors and Affiliations}

Javier Martínez Caballero ${ }^{1}$ (D) . Lucía González González ${ }^{1}$ (D) - Elías Rodríguez Cuéllar ${ }^{1}$ (D) Eduardo Ferrero Herrero ${ }^{1}$ (D) . Cristina Pérez Algar ${ }^{2}$. Victor Vaello Jodra ${ }^{2}$ (D) María Dolores Pérez Díaz ${ }^{3}$. Jana Dziakova ${ }^{4}$ (D)

Rosario San Román Romanillos ${ }^{5}$. Marcello Di Martino ${ }^{6}$ (D) Ángela de la Hoz Rodríguez ${ }^{6}$ (D) Mónica Galán Martín ${ }^{7}$ (D) Daniel Sánchez López $^{8}$ (D) . Mariana García Virosta ${ }^{8}$ (D) Marta de la Fuente Bartolomé ${ }^{(1 D}$. María de Mar Pardo de Lama ${ }^{10}$. María Gutiérrez Samaniego ${ }^{11}$ (D) David Díaz Pérez ${ }^{11}$ D . David Alias Jiménez ${ }^{12}$. Luis de Nicolás Navas ${ }^{13}$. Juan José Pérez Alegre ${ }^{13}$. Javier García-Quijada García ${ }^{14}$. Jenny Guevara-Martínez ${ }^{15}$ (iD . Arantxa Villadoniga ${ }^{15} \cdot$ Roberto Martínez Fernández ${ }^{16}$

1 Digestive and General Surgery Department, 12 de Octubre University Hospital, Madrid, Spain

2 Digestive and General Surgery Department, Ramón y Cajal University Hospital, Madrid, Spain

3 Digestive and General Surgery Department, Gregorio Marañón University Hospital, Madrid, Spain

4 Digestive and General Surgery Department, Clínico San Carlos University Hospital, Madrid, Spain

5 Digestive and General Surgery Department, Príncipe de Asturias University Hospital, Madrid, Spain

6 Digestive and General Surgery Department, La Princesa University Hospital, Instituto de Investigación Sanitaria Princesa (IIS-IP), Universidad Autónoma de Madrid (UAM), Madrid, Spain

7 Digestive and General Surgery Department, Severo Ochoa University Hospital, Madrid, Spain

8 Digestive and General Surgery Department, Infanta Sofía University Hospital, Madrid, Spain
9 Digestive and General Surgery Department, Infanta Elena University Hospital, Madrid, Spain

10 Digestive and General Surgery Department, Fundación Alcorcón University Hospital, Madrid, Spain

11 Digestive and General Surgery Department, Torrejón University Hospital, Madrid, Spain

12 Digestive and General Surgery Department, Rey Juan Carlos University Hospital, Madrid, Spain

13 Digestive and General Surgery Department, Gómez Ulla Defense Central Hospital, Madrid, Spain

14 Digestive and General Surgery Department, Getafe University Hospital, Madrid, Spain

15 Digestive and General Surgery Department, La Paz University Hospital, Madrid, Spain

16 Digestive and General Surgery Department, El Tajo University Hospital, Madrid, Spain 